\title{
Ultrashort Pulsed Laser Induced Heat Affected Zones Characterized by Ion Channeling Contrast Imaging
}

\author{
Heather Thompson ${ }^{1}$, Joel Lammatao ${ }^{1}$, Matthew D. Hecht ${ }^{1}$, Aziz Yousif ${ }^{1}$, Benjamin R. Campbell ${ }^{2}$ and \\ Yoosuf N. Picard ${ }^{1}$ \\ 1. Materials Science and Engineering, Carnegie Mellon University, Pittsburgh, PA, USA. \\ 2. School of Engineering, Mathematics and Science, Robert Morris University, Pittsburgh, PA, USA.
}

Ultrashort pulsed lasers continue to see expanded application in many technologies such as microelectronics and precision manufacturing. The extremely short time duration (picosecond to femtosecond time scales) of these laser pulses create extremely non-equilibrium conditions during the heating and ablation of target materials. The result is generally efficient machining with little to no heat affected zone (HAZ) in the vicinity of ablation. The extent and nature of any possible HAZ continues to be studied by materials characterization methods including electron backscatter diffraction (EBSD) [1] or transmission electron microscopy (TEM) [2]. The effect of pulse duration variation in the ultrashort time scales ( $p$ s to fs) has not been widely studied.

In this study, focused ion beam (FIB) cross-sectioning and ion channeling contrast imaging (ICCI) is employed to characterize the microstructure near ultrashort pulsed laser ablated holes in copper. ICCI can reveal microstructure through orientation contrast. Thus, ICCI can be used to evaluate HAZ by determining if there is any evidence of melting and recrystallization, where consequently the local grain size will be reduced. A FEI Nova Nanolab with a $\mathrm{Ga}^{+}$source operating at $30 \mathrm{keV}$ is used for both cross-sectioning (nA's of current) and imaging (pA's of current). An example cross-section and ICCI micrograph is presented in Figure 1 of the copper specimen free of any laser irradiation. Existing grains in the specimen are tens of microns in size with some twinning evident (rectangular contrast features).

Figure 2(a) and 2(c) present SEM micrographs of ablated holes in the copper specimen by a picosecond laser and femtosecond laser, respectively. A Lumera SUPER RAPID ps laser system operating at 979 $\mathrm{Hz}$ repetition rate generated $10 \mathrm{ps}$ duration laser pulses at $1064 \mathrm{~nm}$ wavelength. A Clark-MXR CPA 2010 fs laser system operating at $979 \mathrm{~Hz}$ repetition rate generated 200 fs duration laser pulses at $775 \mathrm{~nm}$ wavelength (the ps laser was triggered to match the pulse rate of the fs laser). Both laser ablated holes resulted from focused laser irradiation for a $100 \mathrm{~ms}$ exposure. Comparable laser fluences were used to ensure reasonable HAZ size comparisons; $6.4 \mathrm{~J} / \mathrm{cm}^{2}$ and $7.7 \mathrm{~J} / \mathrm{cm}^{2}$ for ps and fs lasers, respectively.

The black dotted rectangles in Figure 2(a) and 2(c) denote the location where FIB cross-sectioning was performed. ICCI micrographs of the FIB cross-sectioned regions are presented in Figure 2(b) and 2(d) where the ablated hole edge is located on the left side of each image. The copper microstructure exhibits typical grain size up to a $\sim 5 \mu \mathrm{m}$ region near the ablated hole edge. This region where the HAZ may be discernible is magnified and presented in Figure 3.

At high magnifications, regions are identifiable where the apparent grain size has been reduced to submicron length scales (denoted by black lines in Figure 3(a) and 3(b)). Lack of porosity indicates this zone is not re-deposition of ablated material. By designating these regions as the HAZ, it appears that the HAZ is smaller for the fs laser than the ps laser. This result would be consistent with long standing trends between HAZ size and pulse duration [3]. However, it is worth noting that appreciable strain is 
evident for the fs laser case. Lines of contrast visible in Figure 3(b) indicate appreciable curvature within copper grains. This curvature indicates significant plastic strain is present within $5 \mu \mathrm{m}$ of the fs laser ablated hole edge. Such strain is not evident for the ps laser case. These results indicate a significant transition in the thermo-mechanical behavior of laser ablation between the ps and fs laser scenarios, a consequence of rapid thermal expansion and mechanical shock induced by sub-ps laser pulses [4]. Trends regarding pulse duration and fluence relationship to HAZ will be reported.

\section{References:}

[1] A. Kumar and T.M. Pollock, Journal of Applied Physics 110 (2011), p. 083114.

[2] J. Bonse and J. Krüger, Journal of Applied Physics 107 (2010), p.054902.

[3] B.N. Chichkov, C. Momma, S. Nolte, F. von Alvensleben and A. Tünnermann, Applied Physics A 63 (1996), p.109.

[4] Q. Feng, Y.N. Picard, H. Liu, S.M. Yalisove, G. Mourou and T.M. Pollock, Scripta Materialia 53 (2005), p. 511.

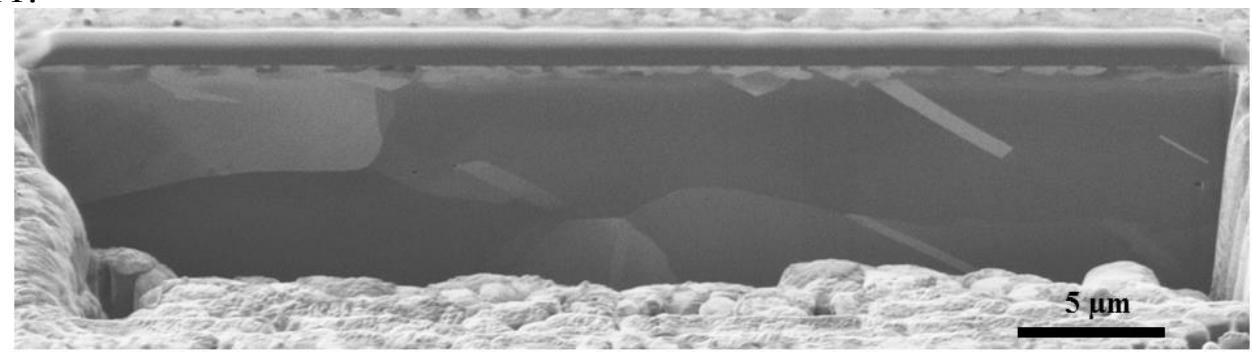

Figure 1. ICCI micrograph of a cross-section in the copper specimen far from any laser irradiation.
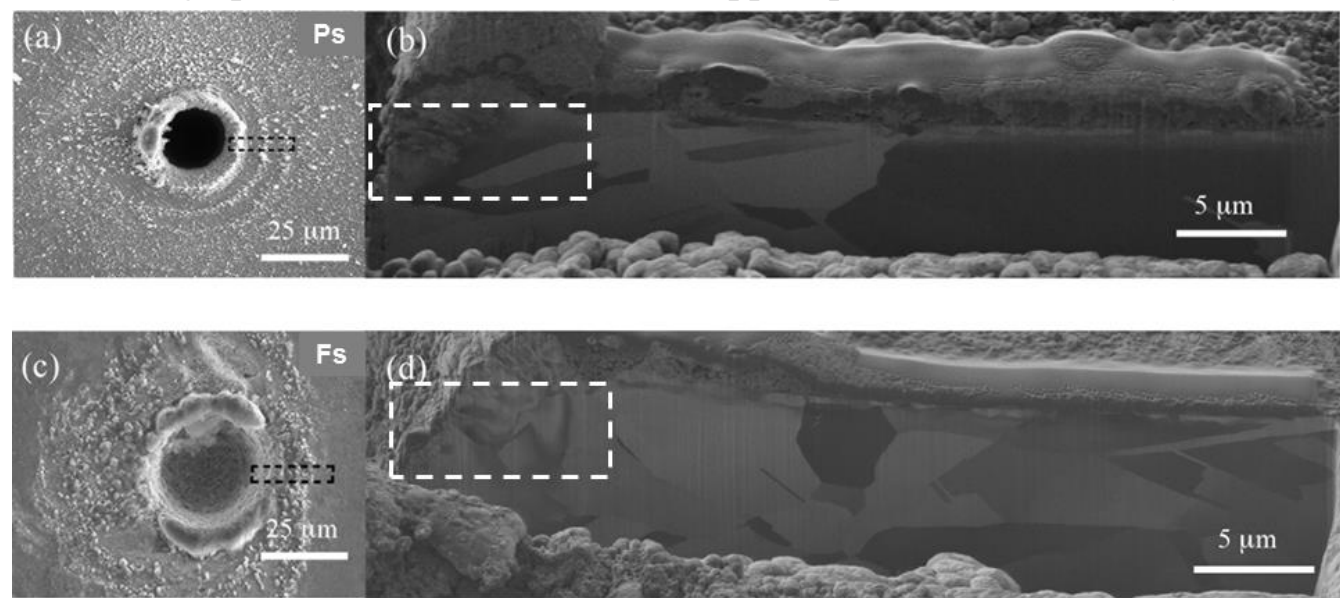

Figure 2. (a) SEM micrograph of a ps laser ablated hole in copper and (b) cross-section ICCI micrograph at the ablated hole edge. (c) and (d) are the same for a fs laser ablated hole. Black rectangles in $(\mathrm{a}, \mathrm{c})$ denote location of cross-sectioning. White rectangles in $(\mathrm{b}, \mathrm{d})$ denote location of Figure 3.
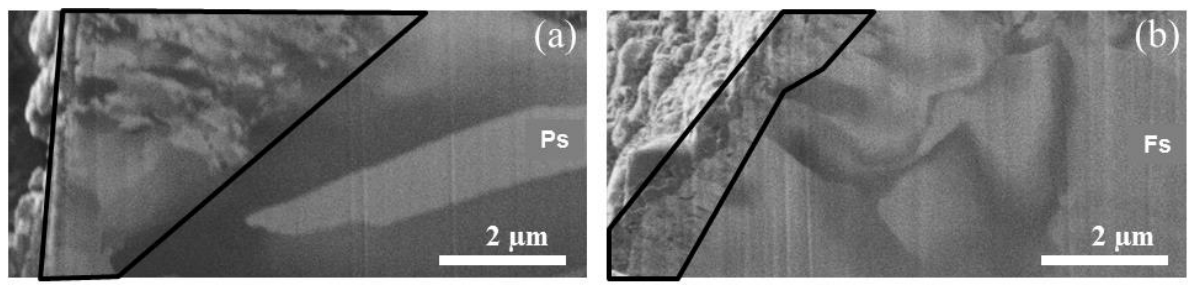

Figure 3. Magnified ICCI micrographs of suspected HAZ (black outlines) for (a) ps laser and (b) fs laser ablated hole edges. 